lustitia Socialis. Revista Arbitrada de Ciencias Jurídicas.

Año V. Vol. V. №3. Edición Especial. 2020-III:

Universidad Regional Autónoma de los Andes

Hecho el depósito de Ley: FA2016000064 ISSN: 2542-3371

FUNDACIÓN KOINONIA (F.K). Santa Ana de Coro, Venezuela

Iván Xavier León-Rodríguez; Cristhian Teobaldo Sedeño-Jiménez

http://dx.doi.org/10.35381/racii.v5i3.1134

\title{
Necesidad de normar la mediación como requisito previo obligatorio en los conflictos de alimentos
}

\section{Need to regulate mediation as a mandatory prerequisite in food disputes}

\author{
Iván Xavier León-Rodríguez \\ us.ivanleon@uniandes.edu.ec \\ Universidad Regional Autónoma de Los Andes, Santo Domingo \\ Ecuador \\ https://orcid.org/0000-0002-3725-988X \\ Cristhian Teobaldo Sedeño-Jiménez \\ us.cristhiansedeno@uniandes.edu.ec \\ Universidad Regional Autónoma de Los Andes, Santo Domingo \\ Ecuador
}

Recibido: 31 de octubre de 2020

Revisado: 10 de octubre de 2020

Aprobado: 05 de diciembre de 2020

Publicado: 10 de diciembre de 2020 
lustitia Socialis. Revista Arbitrada de Ciencias Jurídicas.

Año V. Vol. V. N³. Edición Especial. 2020-III:

Universidad Regional Autónoma de los Andes

Hecho el depósito de Ley: FA2016000064 ISSN: 2542-3371

FUNDACIÓN KOINONIA (F.K). Santa Ana de Coro, Venezuela

Iván Xavier León-Rodríguez; Cristhian Teobaldo Sedeño-Jiménez

\title{
RESUMEN
}

La investigación tiene como objetivo analizar la necesidad de normar la mediación como requisito previo obligatorio en los conflictos de alimentos en el Ecuador. Se desarrolló desde una metodología descriptiva documental con diseño bibliográfico no experimental. En el Centro de mediación de la función Judicial Santo Domingo de los Tsáchilas De los casos atendidos desde enero a diciembre 2017 en casos atendidos 2241, en audiencias instaladas 1242 equivale al 55,42\%, con acuerdos logrados 1152 que representa al $92,75 \%$, en enero a diciembre 2018 , en casos atendidos 20145 , con audiencias instaladas 1156 equivale al 56,53\%. La mediación es un método alterno de solución de conflictos en los juicios de alimentos cuyo procedimiento, genera mayor acceso para descongestionar en forma rápida las causas judiciales en la gestión de justicia y economiza tiempo, dinero, energías en comparación con un juicio.

Descriptores: Mediación; administración de justicia; procedimiento legal. (Palabras tomadas del Tesauro UNESCO).

\begin{abstract}
The objective of the research is to analyze the need to regulate mediation as a mandatory prerequisite in food disputes in Ecuador. It was developed from a descriptive documentary methodology with a non-experimental bibliographic design. In the Mediation Center of the Santo Domingo de los Tsáchilas Judicial function Of the cases attended from January to December 2017 in cases attended 2,241, in hearings installed 1,242 is equivalent to $55.42 \%$, with agreements reached 1,152 which represents $92.75 \%$, in January to December 2018, in 20145 cases attended, with 1,156 hearings installed, equivalent to $56.53 \%$. Mediation is an alternative method of conflict resolution in food trials, the procedure of which generates greater access to quickly decongest court cases in the management of justice and saves time, money, energy compared to a trial.
\end{abstract}

Descriptors: Mediation; administration of justice; legal procedure. (Words taken from the UNESCO Thesaurus). 
lustitia Socialis. Revista Arbitrada de Ciencias Jurídicas.

Año V. Vol. V. N³. Edición Especial. 2020-III:

Universidad Regional Autónoma de los Andes

Hecho el depósito de Ley: FA2016000064

ISSN: 2542-3371

FUNDACIÓN KOINONIA (F.K). Santa Ana de Coro, Venezuela

Iván Xavier León-Rodríguez; Cristhian Teobaldo Sedeño-Jiménez

\section{INTRODUCCIÓN}

El presente artículo ubica la situación conflictiva dentro del Sistema de Justicia Ecuatoriana con la existencia de una elevada carga procesal de trámites en Juicios de alimentos produciendo malestar entre los usuarios por los tiempos y a veces hasta años para la culminación del proceso, de acuerdo a los datos estadísticos brindados por el consejo de la judicatura, para descongestionar ese material procesal tenemos como medio alternativo de resolución de conflictos como es la mediación como lo señala la Constitución de la Republica, siendo un organismo que debe facilitar, ágil y eficiente. La naturaleza y alcance, está en el Estado de Ecuador es el único garante a través de la aplicación de la ley en los Consejos de la Judicatura al beneficiario, al facilitar el acceso a una justicia equitativa en derecho, lastimosamente esto no se cumple en la realidad; ya que existe proceso que tienen dificultad en las áreas de la niñez, civil, transito, penal, dificultando la administración de justicia; sin embargo, esto se puede remediar si se efectúa como medio de solución la mediación y en especial para descongestionar con agilidad, rapidez los trámites en conflictos de alimentos, ahorrando tiempo, recursos a las partes involucradas que aceptan este medio alternativo y por consiguiente al Estado.

El inicio de la mediación se da con el nacimiento de la civilización como método de acuerdo y se estableció con anterioridad en la justicia ordinaria, la aparición se origina como producto de la evolución de justicia por mano propia, cuando la monarquía primitiva hace su aparición, los reyes comienzan a celebrar la actuación de los jueces, por esta razón ya no sería un mediador conocido como tercero neutral si interviene en la solución de controversias.

Los medios alternativos de solución de conflictos se encuentran establecidos en la sección octava inciso primero Art.190 de la Constitución de la República del Ecuador, en la que entre los medios alternativos de resolución de conflicto se reconoce a la mediación como procedimientos alternativos para la solución de controversias siempre y cuando sea con sujeción a la ley y en materias en las que se pueda transigir (Asamblea Nacional, 2008); pero lamentablemente aunque se encuentren consagrado en nuestra 
lustitia Socialis. Revista Arbitrada de Ciencias Jurídicas.

Año V. Vol. V. N³. Edición Especial. 2020-III:

Universidad Regional Autónoma de los Andes

Hecho el depósito de Ley: FA2016000064

ISSN: 2542-3371

FUNDACIÓN KOINONIA (F.K). Santa Ana de Coro, Venezuela

Iván Xavier León-Rodríguez; Cristhian Teobaldo Sedeño-Jiménez

Constitución, los ciudadanos desconocen de estas alternativas que permiten resolver situaciones litigiosas de forma más vertiginosa, economizando tiempo y recursos, de esta manera, evitar procesos judiciales.

La mediación es un proceso no litigioso, en la que un mediador asiste a las partes a negociar para llegar a un resultado que resuelva sus controversias. "Constituye un esfuerzo estructurado para facilitar la comunicación entre los contrarios, con lo que las partes pueden voluntariamente evitar el sometimiento a un largo proceso judicial, con el desgaste financiero y emocional que este conlleva, pudiendo acordar una solución para su problema de forma rápida, y cordial" (Schiffrin, 1996); mientras que el jurista Guillermo Cabanellas de Torres indica que la mediación es la "Participación secundaria en un negocio ajeno, a fin de prestar algún servicio a las partes o interesado (...) en una controversia, conflicto o lucha" (Cabanellas de Torres, 2006, p. 253). Es importante recalcar que, de los dos enunciados anteriores, sobre la conceptualización de mediación, denotamos que tiene la finalidad de dar por terminado un conflicto antes de llegar a instancias judiciales.

De la definición anteriormente expuesta, se puede concluir que en general y específicamente en cada una de las materias transigibles donde se puede proponer como mediación, siendo el método alternativo de solución de conflictos asistidos por un mediador, es así que pueden llegar acuerdos voluntarios y resolver conflictos entre los involucrados, mismos que plantean sus obligaciones y resoluciones para ello firman un acta (Vázquez-Martínez \& Bazán-Mayagoitia, 2019).

Después de unos criterios de distintos autores es de suma importancia analizar cada una de las posiciones teóricas con relación a la mediación, para determinar su aspecto, avance e importancia de la solución alternativa de los conflictos de un estado. Dentro de nuestra legislación ecuatoriana encontramos la figura legal en cuanto al método alternativo que es la mediación, con el ánimo llegar a una solución de conflictos extrajudicialmente (Serrano-Lucero, 2016).

La Ley De Arbitraje y Mediación en su título II, artículo 43 establece: "La mediación es un 
lustitia Socialis. Revista Arbitrada de Ciencias Jurídicas.

Año V. Vol. V. N³. Edición Especial. 2020-III:

Universidad Regional Autónoma de los Andes

Hecho el depósito de Ley: FA2016000064

ISSN: 2542-3371

FUNDACIÓN KOINONIA (F.K). Santa Ana de Coro, Venezuela

Iván Xavier León-Rodríguez; Cristhian Teobaldo Sedeño-Jiménez

procedimiento de solución de conflictos por el cual las partes, asistidas por un tercero neutral llamado mediador, procuran un acuerdo voluntario, que verse sobre materia transigible, de carácter extrajudicial y definitivo, que ponga fin al conflicto" (Ley de arbitraje y mediación, 2006).

Como se puede denotar la Ley de Arbitraje y Mediación define a la mediación como un medio alternativo extrajudicial de la solución de conflictos en materias transigible, asistidos por un tercero llamado mediador; de la misma manera este mismo cuerpo legal en su Art 44, segundo inciso señala: "podrán someterse al procedimiento de mediación que establece la presente Ley, sin restricción alguna, las personas naturales o jurídicas, públicas o privadas, legalmente capaces para transigir." (Ley de arbitraje y mediación, 2006)

La ley de Arbitraje y Mediación, establece claramente la forma con cual se termina el proceso constando el acuerdo total o parcial al que han llegado las partes, esto lo podemos revisar en su artículo 47, cuarto inciso: "El acta de mediación en que conste el acuerdo tiene efecto de sentencia ejecutoriada y cosa juzgada y se ejecutará del mismo modo que las sentencias de última instancia siguiendo la vía de apremio...", (Ley de arbitraje y mediación, 2006) en esta acta constaran las firmas de las partes, así como la del mediador y el acuerdo pactado se elevará a sentencia ejecutoriada y cosa juzgada, sin que se tenga el derecho a ningún recurso con la excepción en menores y alimentos donde se podrá pedir la revisión de la misma; en caso que las partes no tengan un acuerdo se procederá de acuerdo a lo establecido en la misma ley (Serrano-Lucero, 2016).

En los asuntos de menores y alimentos, el acuerdo a que se llegue mediante un procedimiento de mediación, será susceptible de revisión por las partes, conforme con los principios generales contenidos en las normas del Código de la Niñez y Adolescencia y otras leyes relativas a los fallos en estas materias. En el art 11 del código de la niñez y adolescencia, expresa "El interés superior del niño está orientado a satisfacer el ejercicio efectivo del conjunto de los derechos de los niños, niñas y adolescentes ...", (Código de 
lustitia Socialis. Revista Arbitrada de Ciencias Jurídicas.

Año V. Vol. V. N³. Edición Especial. 2020-III:

Universidad Regional Autónoma de los Andes

Hecho el depósito de Ley: FA2016000064

ISSN: 2542-3371

FUNDACIÓN KOINONIA (F.K). Santa Ana de Coro, Venezuela

Iván Xavier León-Rodríguez; Cristhian Teobaldo Sedeño-Jiménez

la niñez y adolescencia, 2003).

Al examinar se considera la necesidad de proteger de forma equitativa los derechos, deberes de niños, niñas y adolescentes, para la aplicación y garantías, este principio sobresale sobre la diversidad étnica y cultural e interpretación de la presente Ley. Ninguno podrá ir en contra de la norma expresa, sin tener en cuenta previamente el testimonio del menor, que esté en condiciones de pronunciar y conseguir su prosperidad íntegra (Rodrígues, 2017).

El artículo 256 del Código de la niñez y adolescencia nos indica los principios rectores que rigen en favor de la niñez y la adolescencia; la administración de justicia especializada de la Niñez y Adolescencia guiará sus actuaciones y resoluciones con estricto apego a los principios, derechos, deberes y responsabilidades que se establecen en el presente Código. Su gestión se inspira, además, en los principios de humanidad en la aplicación del derecho, priorización de la equidad por sobre la ritualidad del enjuiciamiento, legalidad, independencia, gratuidad, moralidad, celeridad y eficiencia. (Còdigo de la niñez y adolescencia, 2003).

El apartado expuesto, me indica que la norma jurídica solo se mediará en materia transigible siempre y cuando no se vulneren derechos, este procedimiento se lo llevará a cabo en los centros de mediación a nivel nacional, tomando en cuenta la ley para lo cual deben estar acreditados por la autoridad competente. En lo referente al principio de servicio a la comunidad en el Código Orgánico de la Función judicial en su inciso segundo del art 17 nos indica: "El arbitraje, la mediación y otros medios alternativos de solución de conflictos establecidos por la ley, constituyen una forma de este servicio público" (Codigo Organico de la Funciòn Judicial, 2009).

Al analizar la base del ordenamiento jurídico en mención con la finalidad de garantizar a través de los servidores públicos, el cumplimiento y la aceptación de la mediación como medio alternativo para la solución de conflictos. En los procesos de mediación se propiciará posibles soluciones a los conflictos, el progreso depende del acatamiento voluntario y no obligado, el pago de pensiones alimenticias, tiene como límite la tabla de 
lustitia Socialis. Revista Arbitrada de Ciencias Jurídicas.

Año V. Vol. V. N³. Edición Especial. 2020-III:

Universidad Regional Autónoma de los Andes

Hecho el depósito de Ley: FA2016000064

ISSN: 2542-3371

FUNDACIÓN KOINONIA (F.K). Santa Ana de Coro, Venezuela

Iván Xavier León-Rodríguez; Cristhian Teobaldo Sedeño-Jiménez

pensiones alimenticias mínimas expedidas por el Consejo Nacional de la Niñez y Adolescencia.

En este sentido, la investigación tiene como objetivo analizar la necesidad de normar la mediación como requisito previo obligatorio en los conflictos de alimentos en el Ecuador.

\section{MÉTODO}

El trabajo investigativo se desarrolló desde una metodología descriptiva documental con diseño bibliográfico no experimental, recopilándose datos documentales y estadísticos en las instituciones relacionadas al tema de investigación, lo cual permitió su análisis a través del método analítico - sintético con la finalidad de interpretar la información, construyéndose una síntesis teórica del objeto de estudio.

\section{RESULTADOS}

Conforme al estudio de campo las estadísticas brindadas por el Consejo de la Judicatura, mediante el oficio -CJ -DNMFJ-2019-0263-0F emitidos el 17 de septiembre del 2019 por parte de la Ab. Catherine Pesantez Villacis, Directora Nacional encargada de la Dirección Nacional de Mediación de la Función Judicial de los casos atendidos en la oficina en el Centro de Mediación desde el 2017, 2018, 2019 y el alcance emitido en derivaciones, se desprende la información desde enero a diciembre del 2017, 2018 y enero a agosto del 2019, en la Provincia de Santo Domingo de los Tsáchilas y Concordia del Centro Nacional de Mediación de la Función Judicial existió un total de 5746 casos atendidos en materia de familia en alimentos, de los cuales 2960 casos lograron acuerdos por el método alternativo de la mediación, equivalente $89,67 \%$; siendo esto un resultado satisfactorio, al ahorrar tiempo y recursos a los usuarios en sus causas y mejorar el descongestionamiento en la administración de Justicia ampliando lo expuesto, se detallan los resultados: 
lustitia Socialis. Revista Arbitrada de Ciencias Jurídicas.

Año V. Vol. V. №3. Edición Especial. 2020-III:

Universidad Regional Autónoma de los Andes

Hecho el depósito de Ley: FA2016000064 ISSN: 2542-3371

FUNDACIÓN KOINONIA (F.K). Santa Ana de Coro, Venezuela

Iván Xavier León-Rodríguez; Cristhian Teobaldo Sedeño-Jiménez

Tabla 1.

Casos de mediación con acuerdos logrados.

\begin{tabular}{lccccc}
\multicolumn{1}{c}{ Periodo } & $\begin{array}{c}\text { Casos } \\
\text { atendidos }\end{array}$ & $\begin{array}{c}\text { Audiencia } \\
\text { Instaladas }\end{array}$ & $\begin{array}{c}\% \text { audiencia } \\
\text { instalada. }\end{array}$ & $\begin{array}{c}\text { Acuerdo } \\
\text { logrado }\end{array}$ & $\begin{array}{c}\text { \% acuerdos } \\
\text { logrados }\end{array}$ \\
\hline $\begin{array}{l}\text { Enero-Diciembre } \\
2017\end{array}$ & 2241 & $\mathbf{1 2 4 2}$ & $55,42 \%$ & $\mathbf{1 1 5 2}$ & $\mathbf{9 2 , 7 5 \%}$ \\
$\begin{array}{l}\text { Enero-Diciembre } \\
2018\end{array}$ & 2045 & $\mathbf{1 1 5 6}$ & $56,53 \%$ & $\mathbf{1 0 1 5}$ & $\mathbf{8 7 , 8 0 \%}$ \\
$\begin{array}{l}\text { Enero -Agosto 2019 } \\
\text { TOTAL }\end{array}$ & 1460 & $\mathbf{0 9 0 3}$ & $61,85 \%$ & $\mathbf{0 7 9 3}$ & $\mathbf{8 7 , 8 2 \%}$ \\
& 5746 & $\mathbf{3 3 0 1}$ & $57,45 \%$ & $\mathbf{2 9 6 0}$ & $\mathbf{8 9 , 6 7 \%}$ \\
\hline
\end{tabular}

Fuente: Dirección Nacional de Mediación de la Función Judicial. Septiembre 2019.

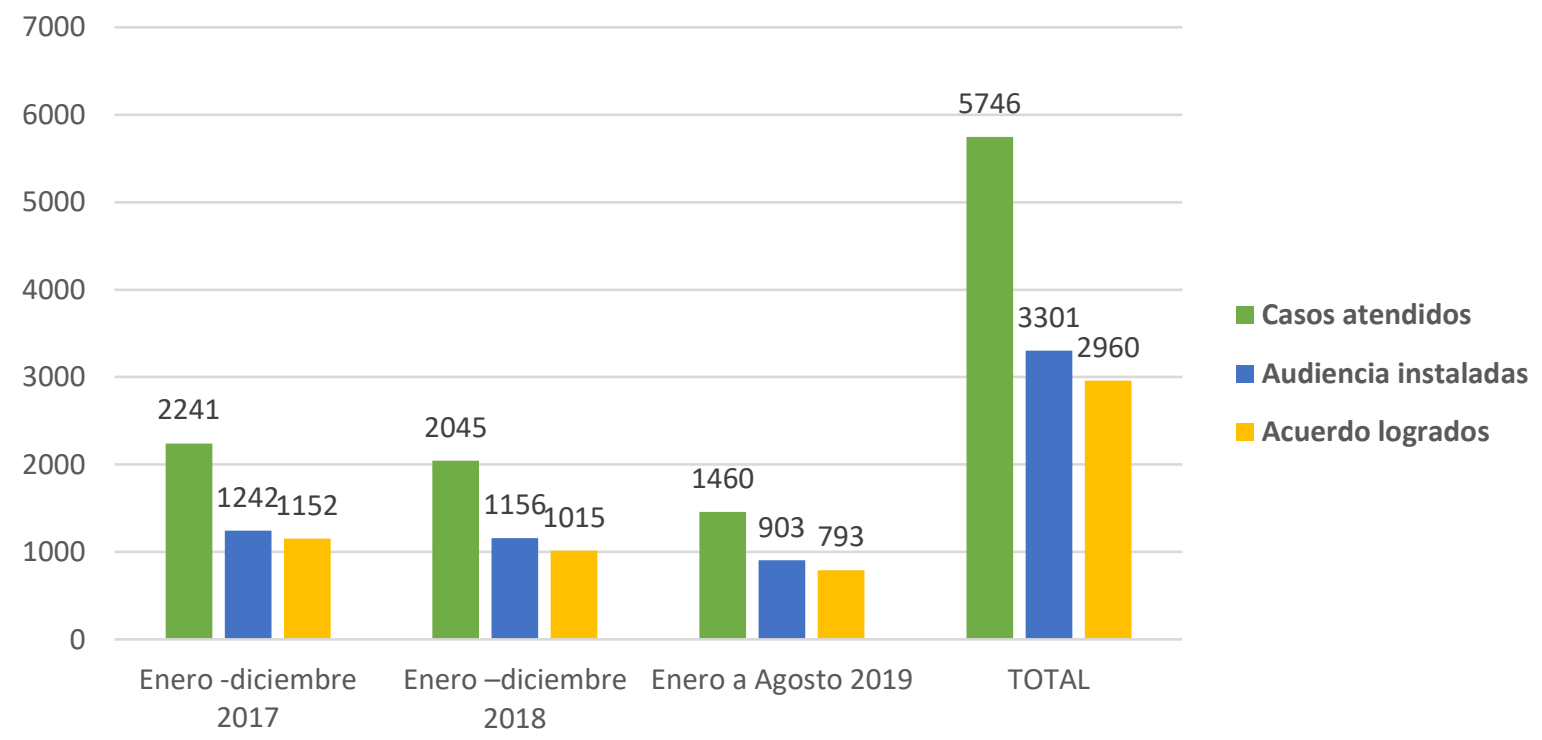

Gráfico 1. Casos de mediación con acuerdos logrados.

Fuente: Dirección Nacional de Mediación de la Función Judicial. Septiembre 2019. 
lustitia Socialis. Revista Arbitrada de Ciencias Jurídicas.

Año V. Vol. V. №3. Edición Especial. 2020-III:

Universidad Regional Autónoma de los Andes

Hecho el depósito de Ley: FA2016000064

ISSN: 2542-3371

FUNDACIÓN KOINONIA (F.K). Santa Ana de Coro, Venezuela

Iván Xavier León-Rodríguez; Cristhian Teobaldo Sedeño-Jiménez

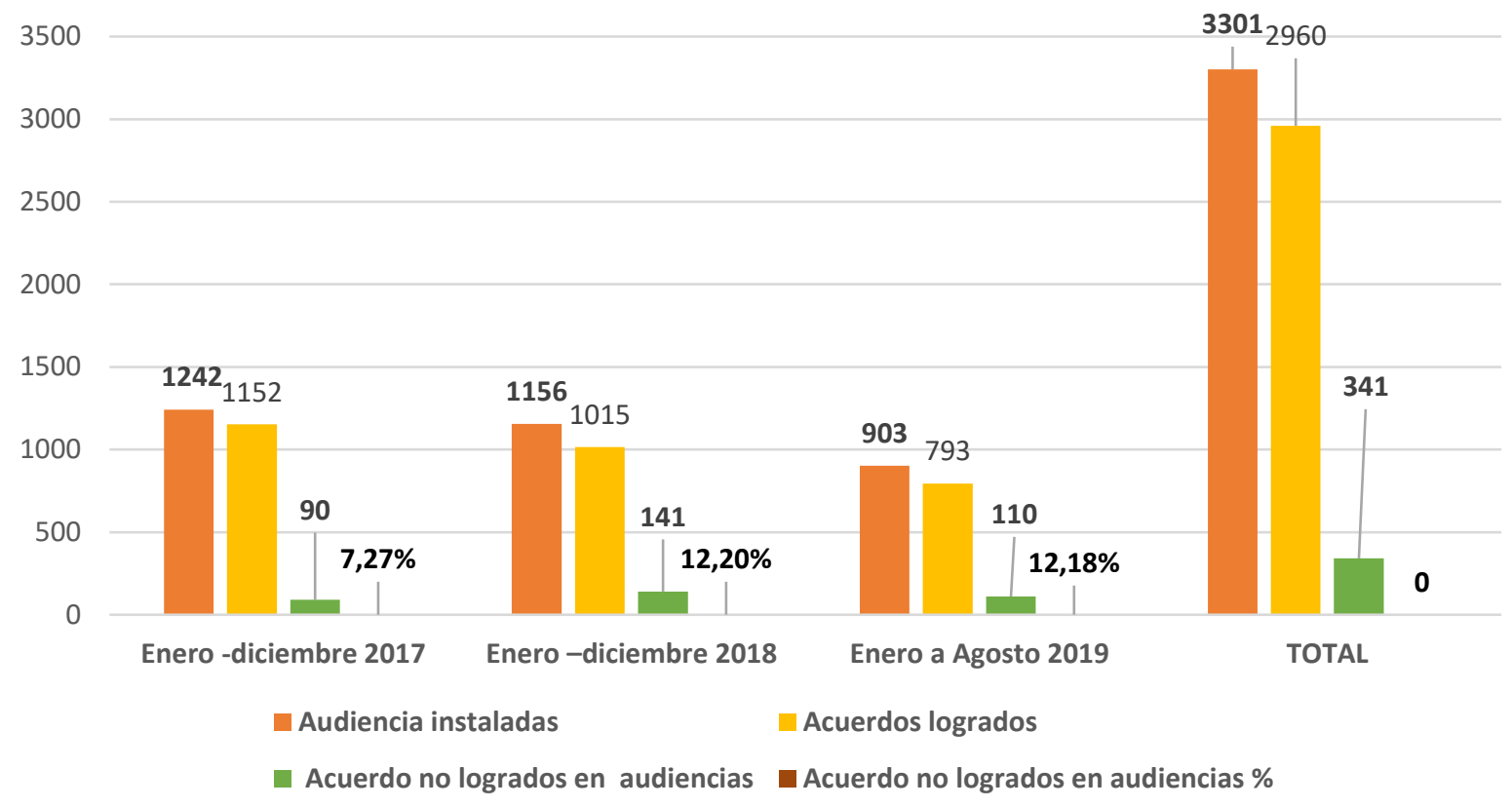

Gráfico 2. Juicios de alimentos con acuerdos logrados a través de la mediación en Centro de mediación.

Fuente: Dirección Nacional de Mediación de la Función Judicial. Septiembre 2019 
lustitia Socialis. Revista Arbitrada de Ciencias Jurídicas.

Año V. Vol. V. N³. Edición Especial. 2020-III:

Universidad Regional Autónoma de los Andes

Hecho el depósito de Ley: FA2016000064 ISSN: 2542-3371

FUNDACIÓN KOINONIA (F.K). Santa Ana de Coro, Venezuela

Iván Xavier León-Rodríguez; Cristhian Teobaldo Sedeño-Jiménez

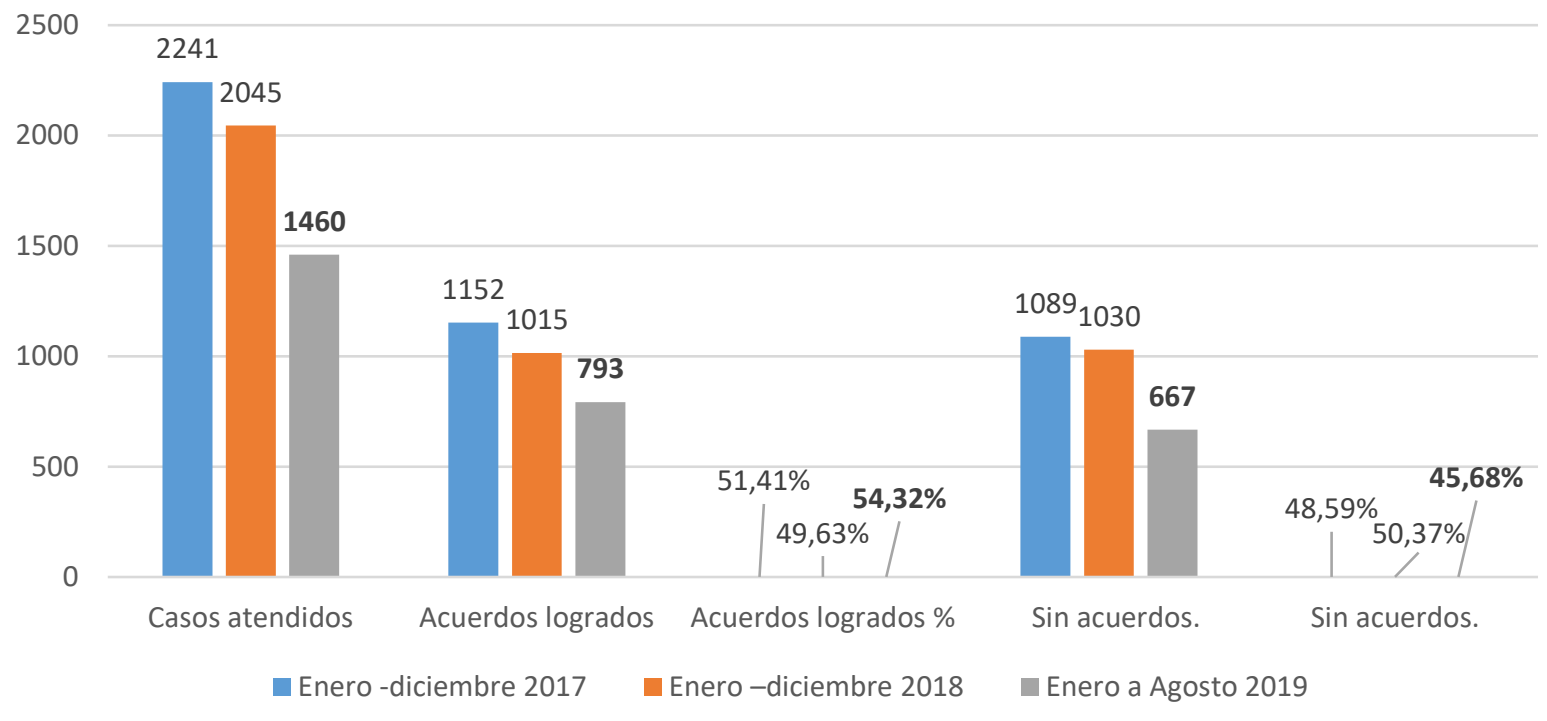

\section{Grafico 3.}

Casos atendidos sin acuerdos en mediación.

Fuente: Dirección Nacional de Mediación de la Función Judicial. Septiembre 2019.

\section{Análisis e interpretación}

Según datos estadísticos de los casos atendidos en la oficina en el Centro de Mediación de la Función Judicial Santo Domingo de los Tsáchilas, en materia en familia de alimentos desde enero 2017-2018 hasta agosto del 2019, un total de 5746 casos, logran acuerdos 2960 casos , equivalente al 89,67\%; en cambio no logran acuerdos unos 341 casos por diferentes factores, que corresponde al 10,33\%; en conclusión más del 89\% en promedio de los tres años de los causas son resueltas, mediante acuerdo a través del método alternativo de la mediación, dando mayor agilidad y mejorando la descongestión en la administración de justicia.

De un total de 3301 de audiencias instaladas, que representa a $51,45 \%$ de los casos atendidos desde enero 2017- 2018 hasta agosto del 2019, logran acuerdos 2960 casos en materia familiar en alimentos y en cambio no logran acuerdos 2786 siendo el $48,69 \%$ 
lustitia Socialis. Revista Arbitrada de Ciencias Jurídicas.

Año V. Vol. V. N³. Edición Especial. 2020-III:

Universidad Regional Autónoma de los Andes

Hecho el depósito de Ley: FA2016000064 ISSN: 2542-3371

FUNDACIÓN KOINONIA (F.K). Santa Ana de Coro, Venezuela

Iván Xavier León-Rodríguez; Cristhian Teobaldo Sedeño-Jiménez

en audiencias instaladas que no logran acuerdos en la oficina en el Centro de mediación de la función Judicial Santo Domingo de los Tsáchilas siendo este dato el total de casos en conflicto en familia en materia de alimentos de los casos atendidos, emitido por la Directora Dirección Nacional de Mediación de la Función Judicial y en Septiembre 2019. En el Centro de mediación de la función Judicial Santo Domingo de los Tsáchilas De los casos atendidos desde enero a diciembre 2017 en casos atendidos 2241, en audiencias instaladas 1242 equivale al 55,42\%, con acuerdos logrados 1152 que representa al $92,75 \%$, en enero a diciembre 2018 , en casos atendidos 20145 , con audiencias instaladas 1156 equivale al 56,53\%, con acuerdos logrados de 1015 representa el $87 \%$ en cambio desde enero a agosto 2019 de 1460 casos atendidos, en audiencias instaladas 903 equivale a $61,85 \%$ y con acuerdos logrados 793 que representa el $87,82 \%$ en conclusión la mayoría de casos en alimento, llegan a acuerdos por mediación más del $87 \%$, siendo este método alternativo el más ágil para la descongestión de causas.

Tabla 4.

Derivaciones de juicios en familia durante desde enero a octubre 2017, 2018 y 2019.

\begin{tabular}{|l|c|c|c|c|c|}
\hline $\begin{array}{l}\text { DERIVACIONES EN } \\
\text { FAMILIA }\end{array}$ & $\begin{array}{c}\text { Casos } \\
\text { atendidos }\end{array}$ & $\begin{array}{c}\text { Audiencias } \\
\text { Instaladas }\end{array}$ & $\begin{array}{c}\text { \%Audiencias } \\
\text { Instaladas }\end{array}$ & $\begin{array}{c}\text { Acuerdos } \\
\text { logrados }\end{array}$ & $\begin{array}{c}\% \text { Acuerdos } \\
\text { logrados }\end{array}$ \\
\hline $\begin{array}{l}\text { MEDIACION SANTO } \\
\text { DOMINGO }\end{array}$ & 129 & 60 & $46,51 \%$ & 54 & $90,00 \%$ \\
\hline $\begin{array}{l}\text { MEDIACION LA } \\
\text { CONCORDIA }\end{array}$ & 7 & 5 & $71,43 \%$ & 5 & $100,00 \%$ \\
\hline TOTAL & 136 & 65 & 47,79 & 2960 & $51,41 \%$ \\
\hline
\end{tabular}

Fuente: Dirección Nacional de Mediación de la Función Judicial. Septiembre 2019. 
lustitia Socialis. Revista Arbitrada de Ciencias Jurídicas.

Año V. Vol. V. №3. Edición Especial. 2020-III:

Universidad Regional Autónoma de los Andes

Hecho el depósito de Ley: FA2016000064 ISSN: 2542-3371

FUNDACIÓN KOINONIA (F.K). Santa Ana de Coro, Venezuela

Iván Xavier León-Rodríguez; Cristhian Teobaldo Sedeño-Jiménez



Gráfico 4. Derivaciones de juicios en familia durante desde enero a octubre 2017, 2018 y 2019.

Fuente: Dirección Nacional de Mediación de la Función Judicial. Septiembre 2019.

\section{Análisis e interpretación}

Durante el periodo comprendido de enero 2017 a octubre 2019, el Centro Nacional de Mediación de la Fundación Judicial a través de su oficina de mediación de la provincia de Santo Domingo de los Tsáchilas atendió un total de 136 casos derivados en materia familia de familia por asunto de alimentos, de los cuales instalaron 65 casos, lo que representa el $47,79 \%$ del total de casos ingresados; de los caso instalados, se logró el $90,77 \%$ en acuerdos sobre las audiencias instaladas, es decir que de cada 6 casos tuvieron imposibilidad de acuerdo. 
lustitia Socialis. Revista Arbitrada de Ciencias Jurídicas.

Año V. Vol. V. №3. Edición Especial. 2020-III:

Universidad Regional Autónoma de los Andes

Hecho el depósito de Ley: FA2016000064

ISSN: 2542-3371

FUNDACIÓN KOINONIA (F.K). Santa Ana de Coro, Venezuela

Iván Xavier León-Rodríguez; Cristhian Teobaldo Sedeño-Jiménez

\section{DISCUSIÓN}

Por lo expuesto de acuerdo a la entrevista con la Ab. Catherine Pesantez Villacis, Directora Dirección Nacional de Mediación de la Función Judicial menciono que en cuanto a las "estadísticas de tiempo y recursos" que toma atender casos en el centro de mediación", de conformidad con el modelo de gestión de las oficinas de mediación, los mediadores deben fijar la audiencia de mediación dentro del plazo máximo de 15 días, a partir de la recepción de la solicitud de audiencia de mediación.

Además, el art 51 de la Ley de Arbitraje y mediación establece: "Si alguna de las partes no comparece a la audiencia de mediación a la que fuere convocada, se señalara fecha para una nueva audiencia. Si en la segunda oportunidad alguna de las partes no compare, el mediador expedirá la constancia de imposibilidad de mediación (...)" (Ley de arbitraje y mediación, 2006). Por otro parte, cada oficina de mediación cuenta con una agenda de atención de audiencias de mediación, dependiendo de la carga existente en cada una de las oficinas de mediación (Mazo-Álvarez, 2014).

Es necesario aclarar que el art 46 de la Ley de Arbitraje y Mediación indica que el resultado de la mediación, en el caso de derivación, debe ser devuelto en el término de 15 días contados desde la recepción por parte del centro, de la notificación del juez. (Ley de arbitraje y mediación, 2006). En consecuencia, el tiempo de atención de un caso en mediación se circunscribirá al plazo y término descritos, y a la agenda de la oficina de mediación, resaltando las características de celeridad y flexibilidad de los procedimientos de mediación (Jequier-Lehuedé, 2016).

Por otra parte, en cuanto a los "recursos" de conformidad con el art 5 del Instructivo de Registro y Funcionamiento de centros de mediación, expedido por el Pleno del Consejo de la Judicatura, mediante Resolución № 026-2018 del 20 de febrero de 2018, para el registro de un centro de mediación se debe cumplir con varios requisitos, entre los cuales constan aquellos referidos en los numerales 3 " Lista actualizada de mediadores" con los respectivos documentos de respaldo. 
lustitia Socialis. Revista Arbitrada de Ciencias Jurídicas.

Año V. Vol. V. N³. Edición Especial. 2020-III:

Universidad Regional Autónoma de los Andes

Hecho el depósito de Ley: FA2016000064

ISSN: 2542-3371

FUNDACIÓN KOINONIA (F.K). Santa Ana de Coro, Venezuela

Iván Xavier León-Rodríguez; Cristhian Teobaldo Sedeño-Jiménez

El Centro Nacional de mediación de la función judicial ha justificado que existen 139 oficinas de mediación a nivel nacional en 115 cantones de las 24 provincias del País la existencia de los recursos humanos o personal, debidamente capacitado para la prestación del servicio de mediación y, recursos administrativos acorde con el modelo de gestión del centro, entre los cuales consta la infraestructura física (lugar en que se va a prestar los servicios), así como los bienes necesarios para este objetivo como son; estación de trabajo, equipo de computación e impresora, área de receptación para los usuarios archivador y sala de audiencia equipada para el desarrollo de las audiencias por lo que los usuarios deberán hacer el uso de acuerdo a sus derechos apegados a la normativa legal.

\section{CONCLUSIONES}

Desde la antigüedad en muchos países la mediación como una forma de solucionar los conflictos alimentarios siendo práctico, ya que solo se necesita la voluntad de las partes y la predisposición; en este procedimiento el mediador quien asiste a las partes para llegar a un acuerdo, evitando la sumisión a un largo proceso judicial, con el desgaste emocional de las partes, si no logran los acuerdos por ello sugiero la mediación como una de la mejor alternativa.

Dar solución a los casos de materia de familia en alimentos es factible a través del método alternativo de mediación por los acuerdos logrados según estadísticas recopiladas durante el análisis investigativo por mediación, debido a la disposición de los compromisos alcanzados, siendo esto la mejor manera por ser democrático para los ciudadanos, accesible sin distinción de ninguna naturaleza, se acomoda a la necesidad, confidencial lo acordado para cada una de las causa, dejando constancia en una acta el arreglo para el cumplimiento en los Centro de Mediación de Santo Domingo de los Tsáchilas dando agilidad en los trámites judiciales.

La mediación es un método alterno de solución de conflictos en los juicios de alimentos cuyo procedimiento, genera mayor acceso para descongestionar en forma rápida las 
lustitia Socialis. Revista Arbitrada de Ciencias Jurídicas.

Año V. Vol. V. N³. Edición Especial. 2020-III:

Universidad Regional Autónoma de los Andes

Hecho el depósito de Ley: FA2016000064

ISSN: 2542-3371

FUNDACIÓN KOINONIA (F.K). Santa Ana de Coro, Venezuela

Iván Xavier León-Rodríguez; Cristhian Teobaldo Sedeño-Jiménez

causas judiciales en la gestión de justicia y economiza tiempo, dinero, energías en comparación con un juicio. De acuerdo a las estadísticas de la Dirección Nacional de Mediación de la Función Judicial de Santo Domingo de los Tsáchilas. Septiembre 2019, el nivel de acuerdos logrados supera el 87\% en los ciclos 2017,2018,2019.

Es primordial conocer la trascendencia que tiene el método alternativo de la mediación en materia de familia en alimentos, sus artículos y normativa legal establecido en la constitución de la República del Ecuador ,tomando en cuenta la voluntad de las partes inmersas en un conflicto, depende el éxito o fracaso en su caso; pero es muy trascendental el vínculo que tiene con otras normas legales en el país, púes uno de los objetivos será conocer las modificaciones importantes que requerirá para su correcto funcionamiento, y de esta manera impulsar a los ciudadanos a que se acerquen a la oficina de mediación para solucionar de manera ágil y llegar a un acuerdo de manera satisfactoria para las partes.

\section{FINANCIAMIENTO}

No monetario.

\section{AGRADECIMIENTO}

A la Universidad Regional Autónoma de los Andes; por motivar el desarrollo de la Investigación.

\section{REFERENCIAS CONSULTADAS}

Asamblea Nacional. (2008). Constitución de la República del Ecuador. Quito-Ecuador. Recuperado de https://n9.cl/sia

Cabanellas de Torres, G. (2006). Diccionario Enciclopedico de derecho usual. Buenos Aires.

Código de la niñez y adolescencia. (2003). (Ley No. 2002-100). Recuperado de https://n9.cl/fokgg 
lustitia Socialis. Revista Arbitrada de Ciencias Jurídicas.

Año V. Vol. V. N³. Edición Especial. 2020-III:

Universidad Regional Autónoma de los Andes

Hecho el depósito de Ley: FA2016000064 ISSN: 2542-3371

FUNDACIÓN KOINONIA (F.K). Santa Ana de Coro, Venezuela

Iván Xavier León-Rodríguez; Cristhian Teobaldo Sedeño-Jiménez

Codigo Organico de la Funciòn Judicial. (2009). Registro Oficial Suplemento 544 de 09mar. -2009. Recuperado de https://n9.cl/wm40

Jequier-Lehuedé, E. (2016). La mediación como alternativa de solución de los conflictos empresariales en Chile: Razones y mecanismos para su regulación [The mediation like an alternative for the solution of enterprise conflicts in Chile. Reasons and mechanisms for his regulation]. Revista de derecho (Valdivia), 29(1), 91118. https://dx.doi.org/10.4067/S0718-09502016000100005

Ley de arbitraje y mediación y sus reformas. Publicada en el Registro Oficial 417 de 14 de diciembre de 2006). Recuperado de https://n9.cl/mjmos

Mazo-Álvarez, H. M. (2014). La mediación como herramienta de la justicia restaurativa [Mediation as a tool for restorative justice]. Opinión Jurídica, 12(23).

Rodrígues, C. (2017). La Mediación. ¿Una respuesta al nuevo paradigma del Derecho? [Mediation. An answer to the new paradigm of Law?]. Revista de Derecho (Universidad Católica Dámaso A. Larrañaga, Facultad de Derecho), (15), 243256. https://dx.doi.org/10.22235/rd.v1i15.1382

Schiffrin, A. (1996). La mediación: aspectos generales [Mediation: general aspects]. Recuperado de https://n9.cl/ih0mw

Serrano-Lucero, C. (2016). Justicia restaurativa: la desatinada prohibición de la mediación penal en los asuntos de violencia de género [Restorative Justice: The Foolish Ban on Criminal Mediation in Gender Violence Matters]. CAPJuridica, 1(1), 147-178.

Vázquez-Martínez, A, \& Bazán-Mayagoitia, N. (2019). Justicia restaurativa y reintegración social: retos procedimentales y estructurales [Restorative Justice and Social Reintegration: Procedural and Structural Challenges]. URVIO Revista Latinoamericana de Estudios de Seguridad, (24), 98113. https://doi.org/10.17141/urvio.24.2019.3789 